\title{
Prevalência de enteroparasitoses em manipuladores de alimentos, Florianópolis, SC
}

\author{
Enteroparasitosis prevalence in food handlers, Florianópolis, SC
}

\author{
Alexandre Costa Nolla ${ }^{1}$ Geny Aparecida Cantos $^{2}$
}

\begin{abstract}
RESUMO
Analisamos 238 amostras fecais oriundas de duas populações de indivíduos na Cidade de Florianópolis, Santa Catarina, Brasil: os que trabalhavam em uma empresa de alimentos e trabalhadores de feiras livres e sacolões. Observou-se que esses manipuladores de alimentos apresentaram elevados índices de parasitose.
\end{abstract}

Palavras-chaves: Manipuladores de alimentos. Parasitas intestinais. Epidemiologia das enteroparasitoses.

\begin{abstract}
We analyzed 238 feces samples in two populations of individuals in the City of Florianópolis, Santa Catarina State, Brazil: we selected workers in a fast food company and workers in street markets and vegetable and fruit markets. The intestinal parasites were high in these food handlers.
\end{abstract}

Key-words: Food handlers. Intestinal parasites. Epidemiology of enteroparasitosis.

A maioria das doenças transmitidas por alimentos está ligada aos hábitos precários de higiene pessoal e doméstica dos manipuladores, à higienização e ao controle ambiental, dentre outros. Algumas maneiras utilizadas para se garantir a qualidade higiênico-sanitária dos alimentos são a realização de programas de educação continuada para os manipuladores de alimentos, a realização semestral de exames parasitológicos desses indivíduos e o fortalecimento do sistema de vigilância sanitária para fiscalização de alimentos oferecidos para a população, incluindo uma legislação adequada. No Brasil, apesar da relevância e da atualidade do problema, são poucos os trabalhos avaliando a ocorrência de enteroparasitoses em manipuladores de alimentos 5 .

Assim, considerando a importância dos manipuladores de alimentos como potenciais transmissores de enteroparasitoses e a possibilidade de interromper este elo na cadeia de transmissão, buscou-se realizar o presente estudo.

Analisou-se amostras fecais de 238 indivíduos sendo 142 do sexo masculino e 96 do sexo feminino durante o período de 2002/2003, na Cidade de Florianópolis SC, Brasil. Esses indivíduos foram distribuídos em dois grupos: A e B. 0 grupo A foi constituído por 119 trabalhadores de uma empresa de alimentos (Fast-food - refeições rápidas), com média de idade de 19 anos. O Grupo B foi de 119 indivíduos que trabalhavam em feiras livres e sacolões, com média de idade de 28 anos.

Pôde-se notar que nas duas populações analisadas 0 parasitismo foi elevado, quando comparado a outros inquéritos parasitológicos ${ }^{4}$. No grupo A, a ocorrência de enteroparasitoses foi de $42,8 \%$ e no grupo B, 47,1\%. Este alto parasitismo também foi analisado em relação às condições sócio-econômicas, sendo que o hábito de comer em casa ou no trabalho não interferiu no grau de parasitismo das populações estudadas. Da mesma forma, pôde-se observar que a maioria dos indivíduos em questão, tanto parasitados como não parasitados, vivia em um ambiente com abastecimento de água tratada, destino adequado dos dejetos e lixo doméstico. Os fatores determinantes do elevado parasitismo foram atribuídos a menor renda familiar, ao número de pessoas residentes em cada domicílio, à escolaridade e ao hábito de ingerir verduras e frutas sem a devida higienização. Quando se levou em consideração o tamanho da família verificou-se que os indivíduos de menor renda e menor escolaridade, eram os mais parasitados. 0 hábito de ingerir maiores quantidades de

\footnotetext{
1. Curso de Pós-Graduação em Farmácia da Universidade Federal de Santa Catarina, Florianópolis SC. 2. Departamento de Análises Clínicas da Universidade Federal de Santa Catarina, Florianópolis SC.

Endereço para correspondência: Dra. Geny Aparecida Cantos. Deptº de Análises Clínicas/CCS/UFSC, Campus Universitário, Caixa Postal 476, 88040-900 Florianópolis, SC.

Tel: $5548331-9919$

e-mail: geny@ccs.ufsc.br

Recebido para publicação em 24/6/2004

Aceito em 12/7/2005
} 
frutas e verduras elevou o parasitismo. Pôde-se notar ainda que os gerentes e subgerentes que trabalhavam em uma empresa de alimentos foram menos parasitados que os cozinheiros, os garçons e as garçonetes.

Em relação às técnicas empregadas é de se considerar que cada parasitose tem a sua peculiaridade, sendo importante que os laboratórios clínicos empreguem diferentes técnicas de concentração na coproscopia parasitológica e que se busque metodologias mais sensíveis para o diagnóstico das enteroparasitoses, de forma a melhorar os resultados obtidos. Os métodos laboratoriais utilizados neste trabalho foram: Lutz, Baermann, Graham (fita adesiva), Faust e o Faust modificado. A análise estatística foi baseada no teste qui-quadrado para tendências, adotando $\mathrm{p}<0,05$ como nível de significância ${ }^{3}$.

Os resultados destas análises estão na Tabela 1 . Nota-se, que a infecção por protozoários foi significativamente mais expressiva que por helmintos nos dois grupos analisados $(\mathrm{p}<0,05)$, sendo que o método de Faust e o de Faust modificado foram os mais sensíveis. 0 método de Lutz foi pouco eficiente para cistos de protozoários e larvas de helmintos, enquanto o método da fita adesiva (método de Graham), foi o mais eficiente para pesquisa de E. vermicularis. Por outro lado, o método de BaermannMoraes detectou maior número de casos de $S$. stercoralis em relação a outras técnicas.

Tabela 1 - Distribuição de enteroparasitas em 238 manipuladores de alimentos da Grande Florianópolis SC, 2002/2003, utilizando diferentes métodos parasitológicos.

\begin{tabular}{|c|c|c|c|c|c|c|c|c|c|c|}
\hline \multirow[b]{2}{*}{ Parasitas } & \multicolumn{2}{|c|}{ Faust } & \multicolumn{2}{|c|}{ Faust mod } & \multicolumn{2}{|c|}{ Lutz } & \multicolumn{2}{|c|}{ Baermann } & \multicolumn{2}{|c|}{ Graham } \\
\hline & $\mathrm{n}^{0}$ & $\%$ & $\mathrm{n}^{0}$ & $\%$ & $\mathrm{n}^{0}$ & $\%$ & $\mathrm{n}^{0}$ & $\%$ & $\mathrm{n}^{0}$ & $\%$ \\
\hline \multicolumn{11}{|l|}{ Helmintos } \\
\hline E. vermicularis & 1 & 0,4 & 1 & 0,4 & - & - & - & - & 11 & 4,6 \\
\hline S. stercoralis & - & - & - & - & - & - & 4 & 1,7 & 0 & 0 \\
\hline A. lumbricoides & - & - & 1 & 0,4 & 4 & 1,7 & - & - & - & - \\
\hline H. nana & 1 & 0,4 & 1 & 0,4 & 1 & 0,4 & - & - & - & - \\
\hline Subtotal & 2 & 0,8 & 3 & 1,3 & 5 & 2,1 & 4 & 1,7 & 11 & 4,6 \\
\hline \multicolumn{11}{|l|}{ Protozoários } \\
\hline E. nana & 42 & 17,6 & 50 & 21,0 & - & - & - & - & - & - \\
\hline B. hominis & 10 & 4,2 & 37 & 15,5 & 1 & 0,42 & - & - & - & - \\
\hline E. coli & 17 & 7,2 & 29 & 12,2 & 7 & 2,9 & - & - & - & - \\
\hline G. lamblia & 9 & 3,8 & 13 & 5,5 & 6 & 2,5 & - & & - & - \\
\hline E. histolytica & 3 & 1,3 & 5 & 2,1 & - & - & - & - & - & - \\
\hline E. hartmanni & 1 & 0,4 & 1 & 0,4 & - & - & - & - & - & - \\
\hline I. butschlii & 1 & 0,4 & 3 & 1,3 & - & - & - & - & - & - \\
\hline Subtotal & 83 & 34,5 & 138 & 58,0 & 14 & 5,9 & & & & \\
\hline Total & 85 & 35,4 & 141 & 59,3 & 19 & 8,0 & 4 & 1,0 & 11 & 4,6 \\
\hline
\end{tabular}

A Tabela 2 mostra a distribuição de enteroparasitas entre os manipuladores de alimentos e verificou-se uma maior prevalência de protozooses do que helmintoses e também frequiências variáveis para cada parasita. De fato, a literatura mostra que este parasito é mais freqüentemente encontrado em crianças com idade de 0 a 5 anos. 0 Blastocystis hominis foi o segundo protozoário de maior ocorrência, seguido de Endolimax nana e Entamoeba coli.

Assim, a utilização de diferentes métodos coproscópicos na rotina de diagnóstico das enteroparasitoses na nossa região ainda se faz necessário. Deve-se salientar que de acordo com os resultados obtidos neste estudo, o método de Faust, na forma tradicional, ainda continua sendo uma boa escolha para o diagnóstico das protozooses intestinais ${ }^{1}$. Contudo, a aplicação de novas metodologias para 0 diagnóstico específico das enteroparasitoses facilita principalmente a determinação da prevalência de portadores assintomáticos, podendo levar ao maior controle das doenças transmitidas pelos mesmos.

Tabela 2 - Distribuição de protozoários e helmintos em 238 amostras fecais de manipuladores de alimentos, grupos A e B, Florianópolis SC, 2002/2003.

\begin{tabular}{|c|c|c|c|c|}
\hline \multirow[b]{2}{*}{ Protozoários } & \multicolumn{2}{|c|}{ Grupo A } & \multicolumn{2}{|c|}{ Grupo B } \\
\hline & $\mathrm{n}^{\mathrm{OA}}$ & $\%$ & $\mathrm{n}^{\mathrm{oB}}$ & $\%$ \\
\hline Endolimax nana & 26 & 21,8 & 26 & 21,9 \\
\hline Blastocystis hominis & 24 & 20,2 & 10 & 8,4 \\
\hline Entamoeba coli & 13 & 10,9 & 22 & 18,5 \\
\hline Giardia lamblia & 1 & 0,8 & 14 & 11,8 \\
\hline Entamoeba histolytica & 1 & 0,8 & 4 & 3,4 \\
\hline Entamoeba hartmanni & 1 & 0,8 & 0 & 0,0 \\
\hline Iodamoeba butschlii & 1 & 0,8 & 2 & 1,7 \\
\hline Enterobius vermicularis & 3 & 2,5 & 8 & 6,7 \\
\hline Strongyloides stercoralis & 2 & 1,7 & 2 & 1,7 \\
\hline Ascaris lumbricoides & 1 & 0,8 & 3 & 2,5 \\
\hline Hymenolepis nana & 1 & 0,8 & 0 & 0,0 \\
\hline Total & 74 & 62,2 & 91 & 76,5 \\
\hline
\end{tabular}

\section{REFERÊNCIAS BIBLIOGRÁFICAS}

1. Cerqueira EJL, Rego, FLT, Rodrigues RC. Avaliação entre métodos baseados na centrifugação em formol éter, utilizados para o diagnóstico de enteroparasitoses. Revista Brasileira de Análises Clínicas 34:107-109, 2002.

2. De Carli GA. Parasitologia clínica. Seleção de métodos e técnicas de laboratório para o diagnóstico de parasitoses humanas. Editora Atheneu, São Paulo, 2002.

3. Guedes MLS, Guedes JS. Bioestatística para profissionais da saúde. Livro Técnico, Conselho Nacional de Desenvolvimento Científico e Tecnológico, Rio de Janeiro p. 201, 1988.

4. Guilherme AL, De Araújo SM, Falavigna DL. Prevalência de enteroparasitas em horticultores e hortaliças da Feira do Produtor de Maringá, Paraná. Revista da Sociedade Brasileira de Medicina Tropical 32:405-411, 1999.

5. Nolla AC, Cantos GA. Ocorrência de enteroparasitas em indivíduos que manipulam alimentos em Florianópolis SC, Brasil. Revista Ciências da Saúde 21:27-31, 2002 . 